\title{
DNA replication is altered in Immunodeficiency Centromeric instability Facial anomalies (ICF) cells carrying DNMT3B mutations
}

\author{
Erica Lana ${ }^{1,2}$, André Mégarbané ${ }^{3,4}$, Hélène Tourrière ${ }^{5}$, Pierre Sarda ${ }^{6}$, Gérard Lefranc ${ }^{5,7}$, Mireille Claustres ${ }^{1,2,6}$ \\ and Albertina De Sario ${ }^{\star, 1,2}$
}

ICF syndrome is a rare autosomal recessive disorder that is characterized by Immunodeficiency, Centromeric instability, and Facial anomalies. In all, $60 \%$ of ICF patients have mutations in the DNMT3B (DNA methyltransferase $3 B$ ) gene, encoding a de novo DNA methyltransferase. In ICF cells, constitutive heterochromatin is hypomethylated and decondensed, metaphase chromosomes undergo rearrangements (mainly involving juxtacentromeric regions), and more than $\mathbf{7 0 0}$ genes are aberrantly expressed. This work shows that DNA replication is also altered in ICF cells: (i) heterochromatic genes replicate earlier in the S-phase; (ii) global replication fork speed is higher; and (iii) S-phase is shorter. These replication defects may result from chromatin changes that modify DNA accessibility to the replication machinery and/or from changes in the expression level of genes involved in DNA replication. This work highlights the interest of using ICF cells as a model to investigate how DNA methylation regulates DNA replication in humans.

European Journal of Human Genetics (2012) 20, 1044-1050. doi:10.1038/ejhg.2012.41; published online 29 February 2012

Keywords: ICF syndrome; DNA replication; DNA methylation

\section{INTRODUCTION}

With the availability of emerging technologies in genomics, a major challenge has become to understand how epigenetic modifications regulate the genome function. Most studies have focused on the relationship between higher-order chromatin structure and gene expression, bringing a breakthrough in the field of gene regulation. In addition to regulating gene expression, epigenetic modifications also contribute to the control of other complex biological processes, such as DNA replication. ${ }^{1}$ Changes in chromatin structure can influence the choice of DNA replication origins during cell differentiation and reprogramming, ${ }^{2}$ determine origin efficiency ${ }^{3}$ and contribute to the establishment of the replication timing. ${ }^{4}$ However, the relationship between epigenetic modifications and DNA replication is far from being fully clarified.

We have used cells from ICF patients (Immunodeficiency Centromeric instability and Facial anomalies, OMIM ID: 242860) to investigate how a DNA methylation defect affects DNA replication in human cells. ICF syndrome is a rare autosomal recessive disorder that is characterized by immunodeficiency, centromeric instability, and facial anomalies. In all, $60 \%$ of patients, called ICF type 1 , have mutations in the DNMT3B (DNA methyltransferase 3B) a de novo DNA methyltransferase that plays a major role during embryo development, but is also active in adult tissues. ${ }^{5}$ The remaining $40 \%$ of patients, either carry mutations in the ZBTB24 transcription factor (ICF type 2) or do not have known mutations. ${ }^{6,7}$ ICF cells are characterized by several molecular defects. Non-coding repetitive sequences (ie, satellites 2 and 3, subtelomeric sequences, and Alu sequences) ${ }^{8-10}$ and genes located in constitutive and facultative heterochromatin (hereafter named C-heterochromatin and F-heterochromatin, respectively) are hypomethylated. ${ }^{11,12}$ This loss of DNA methylation is associated with chromatin decondensation and chromosome instability. ICF-specific changes in RNA levels of genes critical for immune function, development, and neurogenesis were identified through the analysis of global expression profiles. ${ }^{13-16}$ Finally, various late-replicating sequences (ie, satellite 2, telomeric repeats, and genes in F-heterochromatin) replicate earlier in ICF than in control cells. ${ }^{9,11,17}$ In female ICF cells, genes located in F-heterochromatin lose DNA methylation and escape silencing, and this activation process is associated with a change in their replication timing from late to an earlier stage in the Sphase. ${ }^{11}$ It was therefore suggested that the change in the replication timing is responsible for gene activation.

In a previous work, we showed that genes located in C-heterochromatin are hypomethylated and escape silencing in ICF cell lines. ${ }^{12}$ DNA hypomethylation was found in all the analyzed C-heterochromatic genes and in all ICF cell lines, whereas expression was restricted to some genes, every patient having his own group of activated genes. In this work, we asked whether (i) similar to genes located in F-heterochromatin, genes located in C-heterochromatin change their replication timing and (ii) if this change is responsible for aberrant transcription. Moreover, to better understand the relationship between DNA methylation and DNA replication in human cells, we asked whether (iii) DNA hypomethylation modifies the crucial parameters

1INSERM U827, Montpellier, France; ${ }^{2}$ Université Montpellier 1, Montpellier, France; ${ }^{3}$ Unité de Génétique Médicale and Laboratoire Associé INSERM à I'UMR S910, Faculty of Medicine, Saint Joseph University, Beirut, Lebanon; ${ }^{4}$ Institut Jérôme Lejeune, Paris, France; ${ }^{5} \mathrm{CNRS}$ UPR 1142 , Montpellier, France; ${ }^{6} \mathrm{CHRU}$, Montpellier, France; ${ }^{7}$ Université Montpellier 2, Montpellier, France

*Correspondence: Dr A De Sario, INSERM U827, av. du Doyen Gaston Giraud, Montpellier 34096, France. Tel: +33 411759863 ; Fax: +33 411 7598 82; E-mail: albertina.de-sario@inserm.fr

Received 30 November 2011; revised 20 January 2012; accepted 2 February 2012; published online 29 February 2012 
(fork speed, S-phase length, and inter-origin distances) that ensure a complete and correct duplication of the genome. Having wellcharacterized mutations in the DNMT3B gene, ICF patients type 1 are an appropriate model to address this issue in human cells.

\section{MATERIALS AND METHODS}

\section{Lymphoblast cell lines}

ICF and control lymphoblast cell lines have been described previously. ${ }^{12}$ Briefly, ICF3 is a male patient carrying the K770E homozygous mutation. ICF4 is a female patient carrying the D817G homozygous mutation. ICF5 is the Coriell cell line GM8714, a female carrying a heterozygous mutation (A603T/ STP807ins) in the DNMT3B. Lymphoblast cell lines were cultured in RPMI 1640 (Gibco, Invitrogen, Paisley, UK) in the presence of $2 \mathrm{~mm}$ glutamine and $15 \%$ fetal calf serum, at a concentration of 400000 cells per ml. Cell lines were routinely checked for the absence of mycoplasma contamination.

\section{Replication timing}

Exponentially growing cells $\left(\sim 1.5 \times 10^{7}\right.$ cells per time point) were synchronized in G1/S by $20 \mathrm{~h}$ incubation with $0.5 \mathrm{~mm}$ mimosine (no. M0253; Sigma, SaintQuentin Fallavier, France). 5-Bromo-2-deoxyuridine (BrdU) (100 $\mu \mathrm{M}$ ) (no. B5002; Sigma) was added immediately after the release from mimosine. BrdU-containing samples were protected from direct light exposure to avoid BrdU photolysis. Cell samples were collected at $0,4,8,10,12$, and $16 \mathrm{~h}$. The $0 \mathrm{~h}$ sample was collected $15 \mathrm{~min}$ after the addition of BrdU. In total, $3 \times 10^{6}$ cells were used to check cell synchronization and BrdU incorporation using flow cytometry. From the remaining cells, we extracted genomic DNA. Briefly, cells were treated with $100 \mu \mathrm{g} / \mathrm{ml}$ proteinase $\mathrm{K}$, then with $20 \mu \mathrm{g} / \mathrm{ml}$ RNAse, extracted with phenol-chloroform, and finally ethanol precipitated. DNA samples were sonicated in a VibraCell 75022 sonicator (Sonics and Materials, Newtown, CT, USA) to obtain 200-800-bp DNA fragments. For each DNA sample, $75 \mu \mathrm{l}$ of Pan mouse IgG Dynabeads (no. 11041; Invitrogen DYNAL AS, Oslo, Norway) were prebound to $1.5 \mu \mathrm{g}$ of a mouse anti-BrdU IgG1 antibody (clone 3D4; no. 555627; BD Biosciences Pharmingen, San Diego, CA, USA) and then incubated with $2 \mu \mathrm{g}$ of sonicated and denatured DNA. Samples were washed twice with a lysis buffer (50 mм Hepes-KOH (pH 7.5), $140 \mathrm{~mm} \mathrm{NaCl}, 1$ mм EDTA (pH 8.0), $1 \%$ Triton X-100, $0.1 \%$ sodium deoxycholate), twice with a lysis buffer containing $500 \mathrm{~mm} \mathrm{NaCl}$, twice with a wash buffer $(10 \mathrm{~mm}$ Tris- $\mathrm{HCl}(\mathrm{pH}$ 8.0), $250 \mathrm{~mm} \mathrm{LiCl,} 0.5 \%$ NP40, $0.5 \%$ sodium deoxycholate, $1 \mathrm{~mm}$ EDTA ( $\mathrm{pH}$ 8.0)) and once with TE. The immunoprecipitated BrdU-DNA was eluted in $150 \mu$ l of elution buffer (50 mM Tris- $\mathrm{HCl}$ (pH 8.0), $10 \mathrm{~mm}$ EDTA (pH 8.0), 1\% SDS) at $65^{\circ} \mathrm{C}$ for $10 \mathrm{~min}$. The immunoprecipitated DNA was then treated for $2 \mathrm{~h}$ at $37^{\circ} \mathrm{C}$ with $1 \mu \mathrm{g} / \mu \mathrm{l}$ proteinase $\mathrm{K}$, purified with the QIAquick PCR Purification kit (no. 28104; Qiagen, Courtaboeuf, France) and eluted in $40 \mu \mathrm{l}$ of Qiagen EB buffer. Real-time PCR was carried out with a LightCycler 480 (Roche, Mannheim, Germany), using the DNA Master SYBR Green I kit (no. 04707516001; Roche). PCR reactions were carried out in $10 \mu \mathrm{l}$ final volume containing $250 \mathrm{~nm}$ of each primer and $1 / 20$ of the precipitated DNA $(2 \mu \mathrm{l})$. Primers used for quantification are listed in Supplementary Table 1. PCR conditions were the following: $95^{\circ} \mathrm{C}$ for $10 \mathrm{~min}$, followed by 45 cycles of $95^{\circ} \mathrm{C}$ $10 \mathrm{~s}, 60^{\circ} \mathrm{C} 20 \mathrm{~s}$ and $72^{\circ} \mathrm{C} 20 \mathrm{~s}$. Replication timing experiments were carried out in two independent biological, plus two technical replicates. Using real-time PCR, we first quantified each gene in the immunoprecipitated fractions and in the input DNA. We then divided the immunoprecipitated amount of each gene by the corresponding input and we finally subtracted a background noise value, that is, the DNA precipitated from the BrdU-negative sample, corresponding to nonspecifically captured DNA. The calculations are summarized in the following formula: $\left((\mathrm{B} / \mathrm{I})-\left(\mathrm{B}_{\mathrm{BrdU}}{ }_{n e g} / \mathrm{I}_{\mathrm{BrdU}}\right.\right.$ neg $\left.)\right)$, with 'B' for anti-BrdU antibody bound; 'I' for input ( $2 \mu \mathrm{g}$ of sonicated DNA); and 'BrdU_neg' for DNA extracted from cells treated like the other samples, except for the absence of BrdU incubation. The obtained values were normalized representing the last collected point as equal to $100 \%$.

\section{Gene expression}

Total RNA was extracted with the RNAeasy Plus mini kit (no. 74134; Qiagen) according to the manufacturer's recommendations. After extraction, $1 \mu \mathrm{g}$ of total RNA was reverse transcribed using MMLV (no. 28025-013; Invitrogen) and treated with DNAse I (no. 18068-015; Invitrogen) according to the manufacturer's protocol; $1 \mu \mathrm{l}$ of the obtained cDNAs was then used for PCR and $10 \mu \mathrm{l}$ of the reaction were analyzed in ethidium bromide-stained agarose gel. PCR conditions were the following: $95^{\circ} \mathrm{C}$ for $5 \mathrm{~min}$, followed by 50 cycles of $\left(95^{\circ} \mathrm{C}\right.$ $10 \mathrm{~s}, 60^{\circ} \mathrm{C} 20 \mathrm{~s}$, and $72^{\circ} \mathrm{C} 20 \mathrm{~s}$ ). Primers are listed in Supplementary Table 1.

\section{Molecular combing}

Asynchronous exponentially growing cells were subsequently labeled with $25 \mu \mathrm{M}$ 5-iodo-2-deoxyuridine (IdU) (no. I7125; Sigma) for $15 \mathrm{~min}$ and with $200 \mu \mathrm{M}$ 5-chloro-2-deoxyuridine (CldU) (no. 105478; MP Biomedicals, Illkirch, France) for $25 \mathrm{~min}$. After washings, lymphoblasts were mixed with one volume of $1 \%$ pre-heated $\left(42^{\circ} \mathrm{C}\right)$ low-melting point agarose and aliquoted in pulse-field gel electrophoresis molds ( $100 \mu$ l per well) to obtain plugs containing $\sim 1 \times 10^{5}$ cells. Agarose plugs were resuspended in $10 \mathrm{~mm}$ Tris- $\mathrm{HCl}(\mathrm{pH}$ 7.5), $50 \mathrm{~mm}$ EDTA (pH 8.0), $1 \% N$-lauryl sarcosyl (Sigma), and $1 \mathrm{mg} / \mathrm{ml}$ proteinase K (no. P6556; Sigma), and were incubated at $50^{\circ} \mathrm{C}$ for $48 \mathrm{~h}$. Next, plugs were washed four times in $10 \mathrm{ml} \mathrm{TE} \mathrm{(10} \mathrm{mm} \mathrm{Tris} \mathrm{(} \mathrm{pH} 8.0), 1 \mathrm{~mm}$ EDTA) and either stored in this solution at $4{ }^{\circ} \mathrm{C}$ or immediately used for combing. Each agarose plug was melted at $65^{\circ} \mathrm{C}$ for $30 \mathrm{~min}$ in $3 \mathrm{ml}$ of $50 \mathrm{~mm} 4$-morpholinepropanesulfonic acid ( $\mathrm{pH} 5.7$ ), and then treated overnight at $42^{\circ} \mathrm{C}$ with $3 \mathrm{U}$ of $\beta$-agarase no. M0392S; New England Biolabs, Ipswich, MA, USA). The resulting DNA solution was poured into a Teflon reservoir and DNA was combed onto $22 \times 22 \mathrm{~mm}^{2}$ silane-coated glass coverslips (Plateforme de péignage moleculaire, Montpellier, France) as described previously. ${ }^{18}$

\section{IdU, CldU, and DNA detection}

Coverslips with combed DNA were baked for $2 \mathrm{~h}$ at $60^{\circ} \mathrm{C}$, glued onto glass slides, and sequentially dehydrated in $70-90-100 \% \mathrm{EtOH}$ ( 2 min each). DNA was denatured $22 \mathrm{~min}$ in $1 \mathrm{~m} \mathrm{NaOH}$ and the combed slides were subsequently washed five times with $1 \times \mathrm{PBS}$, blocked 15 min with $1 \times \mathrm{PBS} / 0.1 \%$ Triton $\mathrm{X}-100 / 1 \% \mathrm{BSA}$, washed once with PBST $(1 \times \mathrm{PBS} / 0.1 \%$ Triton X-100), and incubated $45 \mathrm{~min}$ at $37^{\circ} \mathrm{C}$ with the following primary antibodies: $1 / 20$ mouse IgG1 anti-BrdU (clone B44; no. 347580; Becton Dickinson, San Jose, CA, USA) for IdU detection and 1/ 20 rat anti-BrdU (clone BU1/75; no. AbC117-7513 AbCys) for CldU detection. Slides were then incubated $30 \mathrm{~min}$ with two secondary antibodies: 1/50 goat antimouse coupled to Alexa 546 and 1/50 goat anti-rat coupled to Alexa 488 (nos. A11030 and A11006; Molecular Probes, Invitrogen, Eugene, OR, USA). After complete detection of IdU and CldU, DNA fibers were counterstained by sequential incubation of the glass slides with $1 / 300$ mouse IgG2a anti-DNA (no. MAB3034; Chemicon, Millipore, Temecula, CA, USA), for $30 \mathrm{~min}$, and 1/50 of goat anti-mouse IgG2a coupled to Alexa 647 (no. A21241; Molecular Probes), for $30 \mathrm{~min}$. Slides were mounted with $20 \mu \mathrm{l}$ of ProLong Gold Antifade Reagent (no. P36930; Molecular Probes, Invitrogen).

\section{Image scanning, signal measurements, and statistical analysis}

Slides were scanned with an epifluorescent microscope (Leica DM6000), using a $40 \times$ objective. Images were recorded with a CoolSNAP HQ1 camera and CldU fluorescent signals were measured with the MetaMorph software (Universal Imaging Corporation, Molecular Devices, Downingtown, PA, USA), using the conversion factor of $340 \mathrm{bp} / \mathrm{pixel} .{ }^{19}$ To calculate the replication fork speed, we measured the CldU tracks, corresponding to the second pulse, and divided the track length by the duration of the pulse. Fork speeds were analyzed by the GraphPad Prism 5 software (GraphPad Software, La Jolla, CA, USA), applying the non-parametric Mann-Whitney two-sample rank-sum test, with a significance level of 0.01 . First, we compared the fork speeds of the control individuals. They showed no significant difference in their fork speed; thus, the data corresponding to each control were pooled. Then, we compared the fork speed of the pool of controls with the fork speed of each ICF patient. To estimate inter-origin distances, we measured the center-to-center distance between adjacent $\mathrm{CldU}$ tracks located on a same fiber (inter-track distance). The measurements were carried out on 200- to 600-kb DNA fibers. Inter-CldUtrack distances were statistically analyzed with the non-parametric MannWhitney two-sample rank-sum test, with a significance level of 0.01 .

\section{Cell cycle analysis}

Exponentially growing cells were synchronized in G1/S by a 20 -h incubation with $0.5 \mathrm{~mm}$ mimosine (no. M0253; Sigma) and harvested every $3 \mathrm{~h}$ after release 
(from time 0 to $15 \mathrm{~h}$ ). BrdU $(10 \mu \mathrm{M})$ was added during the last $15 \mathrm{~min}$ of cell culture. Cells were fixed in cold $70 \%$ ethanol and stored at $-20^{\circ} \mathrm{C}$ until the analysis. All BrdU-containing samples were protected from direct light exposure. For flow cytometry analysis, cells were incubated $30 \mathrm{~min}$ with $50 \mu \mathrm{g} / \mathrm{ml}$ RNAse A at $37^{\circ} \mathrm{C}$, with $2 \mathrm{~N} \mathrm{HCl}$ for $30 \mathrm{~min}$ at room temperature to denature DNA, and then with $0.1 \mathrm{~m}$ borate ( $\mathrm{pH} 8.5$ ) for $2.5 \mathrm{~min}$. BrdU was stained by incubating cells for $1 \mathrm{~h}$ at $37^{\circ} \mathrm{C}$ with $1 / 25$ of mouse anti-BrdU antibody (clone B44; no. 347580; BD Pharmingen, Erembodegem, Belgium), followed by $1 \mathrm{~h}$ incubation at $37^{\circ} \mathrm{C}$ with $1 / 50$ of 5(6)-fluorescein-isothiocyanate (FITC)-conjugated goat anti-mouse antibody (no. 115-095-062; Jackson ImmunoResearch, West Grove, PA, USA ) as secondary reagent. For genomic DNA staining, cells were incubated with $25 \mu \mathrm{g} / \mathrm{ml}$ propidium iodide (no. P4170; Sigma) for $20 \mathrm{~min}$. Fluorescence was recorded on a FACSCaliburII flow cytometer (BD Biosciences) and the cell cycle distribution was analyzed using the FlowJo V.9 software (TreeStar Inc., Ashland, OR, USA) and the Flowing Software 1.6.0 (Cell Imaging Core, Turku Center for Biotechnology, Turku, Finland). At least three biological replicates per cell line were carried out.

\section{RESULTS}

Genes located in C-heterochromatin replicated earlier in ICF cells First, we asked if (i) genes located in C-heterochromatin change their replication timing in ICF cells and if (ii) this change is related to aberrant transcription. We analyzed five genes located in different chromatin domains (Figure 1a). TPTE and TPTE2 are paralogous genes that map to C-heterochromatin in chromosomes 21 and 13, respectively. Both genes are exclusively expressed in the testis and are silent in somatic tissues, including peripheral blood and lymphoblast cell lines. In cancer and ICF cells, these genes lose DNA methylation and escape silencing. ${ }^{12,20}$ STCH and SAMSN-1 are euchromatic genes located in the domain that flanks C-heterochromatin in the long arm of chromosome 21. Both genes are highly expressed in peripheral blood and lymphoblast cell lines and their promoters are associated with histone marks that are specific of an open chromatin. ${ }^{12}$ In addition to these four juxtacentromeric genes, we analyzed CAV2, a euchromatic gene located in $7 \mathrm{q} 31.1$ that was silent in all lymphoblast cells. To assess the replication timing, we immunoprecipitated BrdU-labeled DNA and quantified the bound fraction by real-time PCR (Supplementary Table 2). The analysis was carried out on lymphoblast cell lines derived from three ICF patients and two controls. Cells were synchronized at the G1/S boundary using mimosine, and then released and labeled with BrdU incorporation.

The normal lymphoblast cell lines had very similar replication profiles. Euchromatic genes (CAV2, STCH, and SAMSN-1) had completely replicated by $10 \mathrm{~h}$ after release, whereas C-heterochromatic genes replicated later (Figure 1b). TPTE2 replicated in mid to late S-phase: it started to be synthesized between 4 and $8 \mathrm{~h}$ and continued to be replicated in the following fractions, as indicated by the increase of immunoprecipitated DNA. TPTE replicated late in the S-phase: synthesis started between 8 and $10 \mathrm{~h}$ and replication was still detected between 12 and $16 \mathrm{~h}$.

Next, we compared the controls with each ICF cell line and found that the replication timing of euchromatic genes was preserved, whereas both heterochromatic genes initiated replication at least $2 \mathrm{~h}$ earlier in the ICF cell lines (Figure 1b). TPTE2 was already detectable at $4 \mathrm{~h}$ and had fully replicated by $10 \mathrm{~h}$. The replication timing of TPTE shifted from late to mid S-phase (initiating in the $4-8 \mathrm{~h}$ fraction). When we compared replication timing and gene transcription, we realized that C-heterochromatic genes replicated earlier in all ICF cell lines, but escaped silencing only in some of them (Figure 1b). Altogether, these data show that genes located in C-heterochromatin replicated earlier in the S-phase in ICF cells and that the change of
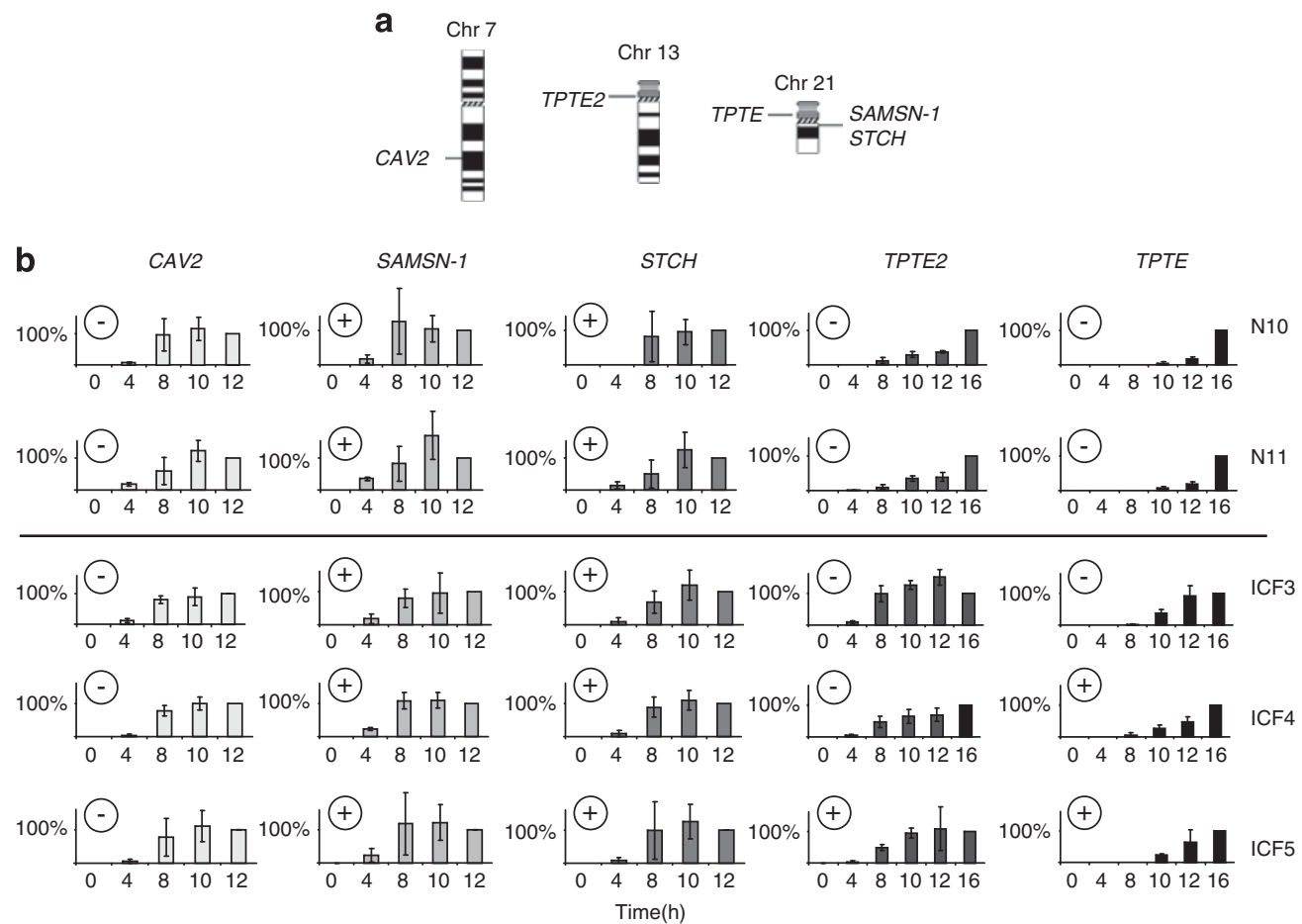

Figure 1 (a) Physical map of two heterochromatic genes, TPTE and TPTE2, and three euchromatic genes, STCH, SAMSN-1, and CAV2. (b) Replication timing in two controls (top) and in three ICF cell lines (bottom). $x$ axis: time (h) of sample collection after the release in the S-phase; $y$ axis: immunoprecipitated DNA divided by the corresponding input and represented as relative percentage of the last collected point (considered equal to $100 \%$ ). Bars are means of 3-4 experiments with standard deviations. Plus and minus refer to gene expression in each cell line (data from ref. 12, except for CAV2, whose expression was analyzed in this work). 
replication timing was not always associated with aberrant transcription. The replication timing was preserved in euchromatic genes, including those located in juxtacentromeric euchromatin.

\section{Global replication fork speed was increased in ICF cells}

Next, to determine if DNA hypomethylation affects the DNA replication kinetics on the whole genome, we measured fork speed by molecular combing in ICF cells. Asynchronous exponentially growing lymphoblast cell lines were sequentially pulse-labeled with IdU and CldU and the incorporated DNA analogues were detected by immunostaining of the combed fibers (Supplementary Figure 1). Replication fork speeds in the controls were very similar (Table 1 and Figure 2), with an overall median value of $1.59 \mathrm{~kb} / \mathrm{min}$ and no significant difference among the three cell lines. We therefore pooled them together and compared each ICF cell line to this pool. Two ICF cell lines (ICF3 and ICF4) showed a highly significant increase in fork speed when compared to the pool of controls, with a median value of 1.95 and $1.98 \mathrm{~kb} / \mathrm{min}$, respectively (Figure 2). In contrast, the fork speed of a third ICF cell line (ICF5) was similar to that of the controls.

Table 1 Analysis of combed DNA fibers in ICF and control lymphoblast cells

\begin{tabular}{lcccccc}
\hline & $N 9$ & $N 10$ & $N 11$ & ICF3 & ICF4 & ICF5 \\
\hline $\begin{array}{l}\text { Fork speed (kb/min) } \\
\quad \text { Median }\end{array}$ & 1.59 & 1.54 & 1.66 & 1.95 & 1.98 & 1.53 \\
Mean & 1.64 & 1.65 & 1.68 & 2.02 & 1.95 & 1.54 \\
SD & 0.61 & 0.80 & 0.48 & 0.86 & 0.72 & 0.57 \\
No. & 151 & 124 & 85 & 145 & 83 & 110 \\
& & & & & & \\
Inter-CldU-track distances $(\mathrm{kb})$ & & & & & & \\
Median & 124.2 & 96.1 & 89.2 & 107.5 & 129.5 & 98.5 \\
Mean & 158.6 & 141.3 & 120.0 & 129.1 & 132.7 & 116.8 \\
SD & 92.6 & 110.5 & 80.8 & 76.9 & 65.5 & 79.6 \\
No. & 38 & 23 & 39 & 25 & 39 & 46 \\
Median fiber length (kb) & 409.7 & 399.3 & 325.0 & 289.7 & 323.7 & 334.6 \\
\hline
\end{tabular}

Abbreviations: $\mathrm{N}$, controls; no., number of measurements; SD, standard deviation.

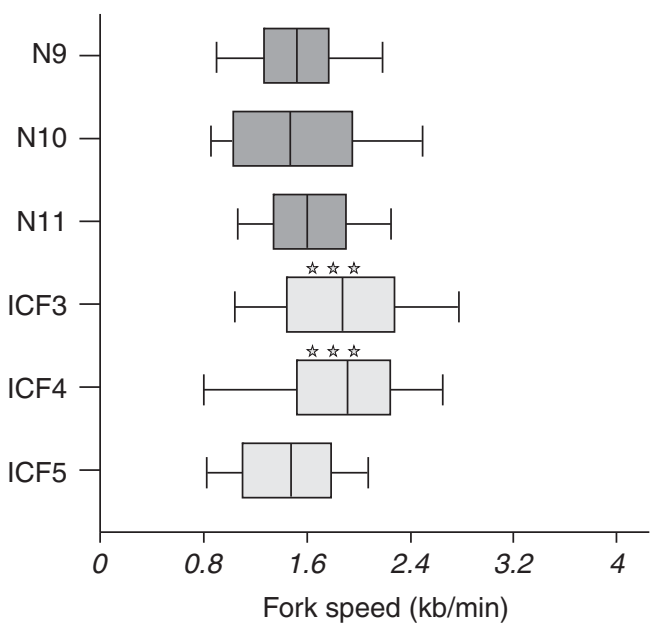

Figure 2 Fork speed is higher in ICF3 and ICF4 patients than in controls. Box plots represent fork speed ( $\mathrm{kb} / \mathrm{min}$ ) in ICF and control (N) cell lines. Median values are indicated by vertical lines in the boxes. Box: 25-75 percentile range; whiskers: 10-90 percentile range. ${ }^{* * *}$ Mann-Whitney rank-sum test: $P$-value $<10^{-4}$.
Overall, the median fork speeds measured in ICF and control lymphoblast cell lines were within the range measured in other human cell lines. ${ }^{21,22}$ The fork speed population showed a broad distribution of values ranging from about 0.4 up to $6 \mathrm{~kb} / \mathrm{min}$, suggesting that different regions of the genome displayed different fork speed. In ICF3 and ICF4, the distribution of the replication fork speed was shifted towards higher values, indicative of a significant increase of replication fork speed in these cells, whereas the speed distribution profile of ICF5 largely resembled the control profile (Supplementary Figure 2). Changes in replication fork speed may be associated with compensatory changes of inter-origin distances. ${ }^{22,23}$ To check whether the increase in fork speed in ICF3 and ICF4 was associated with a change of the inter-origin distance, we measured the center-to-center distance between two adjacent CldU tracks (inter-track distance). This measurement is currently used to estimate inter-origin distance. ${ }^{19}$ To avoid a bias due to the variable fiber length distribution, we analyzed DNA fibers ranging from 200 to $600 \mathrm{~kb}$ and we observed that inter-track distances were similar in ICF and control cell lines (Table 1). To conclude, in two ICF cell lines out of three, replication fork speed was higher compared with that measured in controls. The increase in fork speed was not associated with an increase of the inter-origin distance.

ICF cells progressed faster through the S-phase

Having observed a detectable and significant increase in the global replication fork speed in two ICF cell lines, we asked whether the S-phase length was also altered. We first synchronized cells at the G1/S boundary by incubation with mimosine. After release, we collected cells at six different time points during the S-phase, starting from 0 to $15 \mathrm{~h}$ at 3 -h intervals. This time course allowed lymphoblast cells to re-enter the cell cycle and fully complete the S-phase after recovery from the block. For each of the six time points, we pulsed the cells with BrdU during the last $15 \mathrm{~min}$ of culture, to label the replicating DNA, and we analyzed them by flow cytometry. To investigate ICF-specific changes in the S-phase progression, we directly compared the flow cytometry profiles obtained from ICF and control lines. We considered both replicating DNA (BrdU-labeled) (Figure 3) and total DNA (Supplementary Figure 3) profiles. At least three independent experiments were carried out per cell line. ICF and control cells entered the S-phase and initiated DNA synthesis at the same time after release from the mimosine block, as both replicating DNA and total DNA profiles at $0-3 \mathrm{~h}$ post-release were undistinguishable. Starting from $6 \mathrm{~h}$ post-release, we observed ICF-specific differences: the BrdU-DNA profiles of ICF3 and ICF4 cells at 6 and $9 \mathrm{~h}$ were comparable with the profiles of the controls at 9 and $12 \mathrm{~h}$, respectively (Figure 3). This shift suggested that the two ICF cell lines had progressed faster through the S-phase. Similar results were obtained by observing the total DNA content profiles (Supplementary Figure 3B). ICF5 displayed a cell-cycle profile similar to the profile of the control cell lines. Interestingly, the absence of altered S-phase length in ICF5 was also reported by Yehezkel et al..$^{9}$ Altogether, these data suggested that the S-phase was shorter in the ICF cell lines (two out of three) that had displayed an increased replication fork speed.

\section{DISCUSSION}

In a previous study, we showed that genes located in C-heterochromatin lost DNA methylation and escaped silencing in ICF lymphocytes and cell lines. ${ }^{12}$ The aberrant activation of these genes was variable, each patient having his own group of expressed genes. Herein, we have provided evidence that at least two of these C-heterochromatic genes replicated earlier in the S-phase in ICF cell 


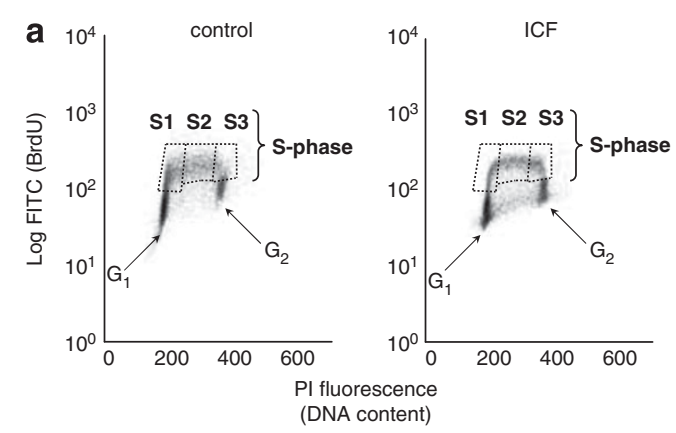

b b

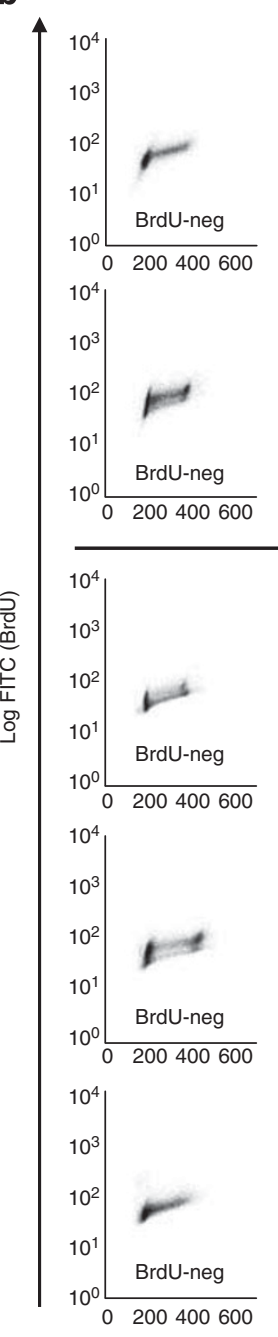

s

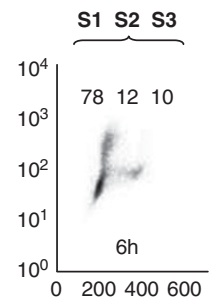

$10^{4}$
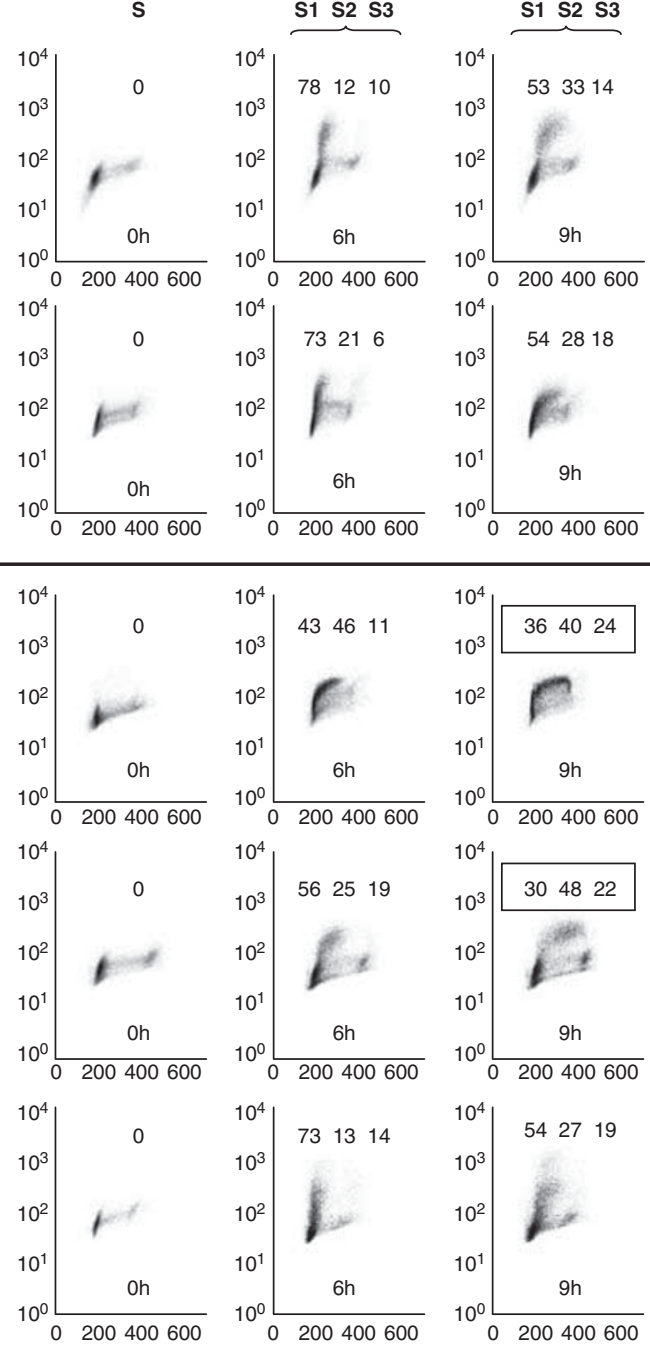

$10^{4}$
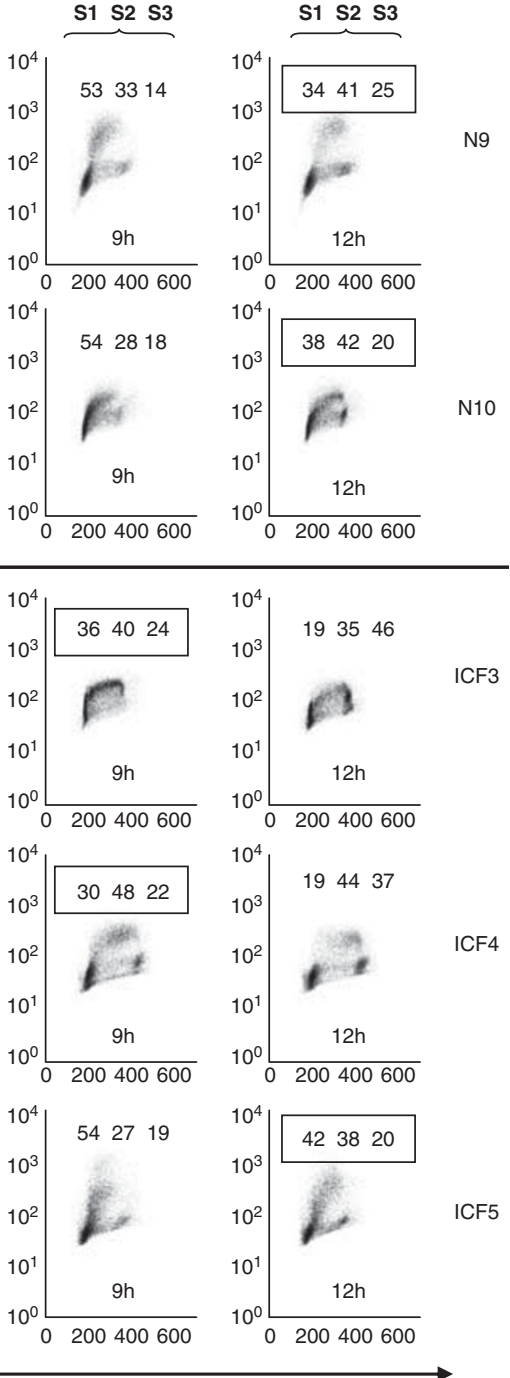

PI fluorescence (DNA content)

Figure 3 Flow cytometry analysis of the S-phase progression: BrdU/propidium iodide (PI) profiles. BrdU was added during the last 15 min of cell culture (excepted for the BrdU-negative samples) and detected by a fluorescent immunostaining. (a) Asynchronous lymphoblasts. $x$ axis: DNA content (PI fluorescence); $y$ axis: BrdU content (FITC fluorescence, logarithmic scale). Cell count is displayed as a density gradient. The three main groups of dots correspond to cells in G1 (lower left), S (upper middle), and G2 (lower right). The window used for the S-phase cell quantification is shown. (b) Flow cytometry profiles of a BrdU-negative sample and of BrdU-labeled samples at 0, 6, 9 and $12 \mathrm{~h}$ post-release, in control and ICF lymphoblast cell lines. The three values on the top of each graph indicate the percentage of cells in S1, S2, and S3 subsets in which the S-phase was divided (S-phase is considered equal to 100\%). Similar distributions (highlighted by rectangles) are observed between the $9 \mathrm{~h}$ profile of ICF3 and ICF4 and the $12 \mathrm{~h}$ profile of controls (N9 and N10) and ICF5. One representative experiment for each cell line is shown.

lines. Our work is consistent with previous results that showed that other late-replicating sequences, namely satellite 2, telomeric sequences, and genes located in F-heterochromatin, replicated earlier in the S-phase in ICF cell lines. ${ }^{9,11,17}$ However, in contrast to genes located in F-heterochromatin, ${ }^{11}$ altered replication timing was not always correlated with activation of the repressed genes: C-hetero- 
chromatic genes replicated earlier in all the analyzed ICF cell lines, but escaped silencing only in some of them. ${ }^{12}$ A correlation between loss of DNA methylation and changes in replication timing has also been reported in imprinted genes. ${ }^{24}$ In 5 -azacytidine-treated cell lines, the relative replication timing of both late- and early-replicating alleles was advanced in imprinted genes; on the contrary, in cell lines treated with histone acetylation inhibitors, the late-replicating allele had a bigger shift in replication timing than the early allele, and this resulted in loss of replication imprinting in the gene. Overall, these data show that DNA methylation and/or chromatin condensation are required for late replication in mammal cells.

Late replication in eukaryotes is settled during development. At the beginning, embryonic cells undergo rapid cycles of cell division to increase the cell number: at this early stage, the S-phase is very short and the whole genome replicates almost simultaneously. In Drosophila, late-replicating sequences appear at the mid-blastula transition, when satellite sequences acquire heterochromatic features. ${ }^{4}$ Similar data are not available in humans; however, this study and others ${ }^{9,11,17}$ have shown that the DNMT3B activity is required to preserve late-replicating sequences. Since this protein starts to be expressed by 6-day epiblasts in the mouse, ${ }^{25}$ it may contribute to settling late replication during development.

ICF cells derived from patients bearing DNMT3B mutations were then used to study how DNA methylation affects replication kinetics. Interestingly, fork speed was significantly higher and S-phase length was shorter in two ICF cell lines out of three. Changes in replication fork speed may be associated with changes of inter-origin distances; ${ }^{22,23}$ however, this did not occur in ICF cell lines that had inter-origin distances comparable to controls. A DNA replication defect generalized to the whole genome was unexpected considering that major epigenetic changes in ICF patients occur in heterochromatic regions (reviewed by Ehrlich $e a^{26}$ ) and that the shift of replication timing is restricted to heterochromatic regions. Two non-mutually exclusive hypotheses can account for the increased fork speed in ICF cell lines. First, as a direct consequence of hypomethylation, DNA may be more accessible to proteins involved in replication because of an open chromatin that facilitates the diverse steps of the replication process. Second, the increased fork speed may result from dysregulation of genes involved in DNA replication. Consistent with the last explanation is the fact that only two ICF cell lines out of three had a significant change in DNA replication kinetics. A similar variability was found in gene expression patterns, each patient having his own subset of dysregulated genes. ${ }^{12-15}$ This variability reflects the heterogeneity of DNMT3B mutations and the resulting phenotypic and molecular variability that is observed among patients. Depending on the residual activity of the DNMT3B protein, each patient has his own transcriptome, and hence may have a different efficiency of DNA replication.

A hallmark feature of ICF syndrome is chromosome instability, mainly involving C-heterochromatin. ${ }^{5}$ Chromosome instability in ICF syndrome has always been associated with hypomethylation of the involved chromosomal regions. ${ }^{27}$ However, it is tempting to suggest that altered DNA replication may also contribute to the genomic instability that characterizes ICF cells, similar to what is observed in other pathological conditions (ie, cancer, ${ }^{28}$ Bloom syndrome, ${ }^{29}$ Rothmund-Thomson syndrome $\mathrm{e}^{30}$ ) where the alteration of the replication process is associated with a high level of spontaneously occurring and/or induced chromosome abnormalities.

This work has provided new insight into the molecular defects that characterize ICF cells showing that not only do DNA hypomethylation, chromosome instability, and transcriptomic changes occur, but also DNA replication is altered. Moreover, this study has shown the interest of using ICF cells as a model to investigate how DNA methylation regulates the DNA replication process in human cells.

\section{CONFLICT OF INTEREST}

The authors declare no conflict of interest.

\section{ACKNOWLEDGEMENTS}

We thank Christelle Cayrou and Philippe Pasero for useful discussions and Melanie Ehrlich and Domenico Maiorano for critical reading of the manuscript. We acknowledge the Montpellier DNA Combing Facility for providing the silanized surfaces. This work was supported by grants of the Fondation Jérôme Lejeune and the Fondation pour la Recherche Médicale to AD, and a grant from the Saint Joseph University to AM.

1 Hiratani I, Gilbert DM: Replication timing as an epigenetic mark. Epigenetics 2009; 4: 93-97.

2 Cayrou C, Coulombe P, Méchali M: Programming DNA replication origins and chromosome organization. Chromosome Res 2010; 18: 137-145.

3 Wu PY, Nurse P: Establishing the program of origin firing during $S$ phase in fission yeast. Cell 2009; 136: 852-864.

4 Shermoen AW, McCleland ML, O'Farrell PH: Developmental control of late replication and S phase length. Curr Biol 2010; 20: 2067-2077.

5 De Sario A: Clinical and molecular overview of inherited disorders resulting from epigenomic dysregulation. Eur J Med Genet 2009; 52: 363-372.

6 de Greef JC, Wang J, Balog J et al: Mutations in ZBTB24 are associated with immunodeficiency, centromeric instability, and facial anomalies syndrome type 2 . Am J Hum Genet 2011; 88: 796-804.

7 Chouery E, Abou-Ghoch J, Corbani S et al: A novel deletion in ZBTB24 in a Lebanese family with immunodeficiency, centromeric instability, and facial anomalies syndrome type 2. Clin Genet 2011; e-pub ahead of print 5 October 2011; doi: 10.1111/ j.1399-0004.2011.01783.x.

8 Jeanpierre M, Turleau C, Aurias A et al: An embryonic-like methylation pattern of classical satellite DNA is observed in ICF syndrome. Hum Mol Genet 1993; 2: 731-735.

9 Yehezkel S, Segev Y, Viegas-Péquignot E, Skorecki K, Selig S: Hypomethylation of subtelomeric regions in ICF syndrome is associated with abnormally short telomeres and enhanced transcription from telomeric regions. Hum Mol Genet 2008; 17: 2776-2789.

10 Miniou P, Bourc'his D, Molina Gomes D, Jeanpierre M, Viegas-Péquignot E: Undermethylation of Alu sequences in ICF syndrome: molecular and in situ analysis. Cytogenet Cell Genet 1997; 77: 308-313.

11 Hansen RS, Stöger R, Wijmenga $\mathrm{C}$ et al: Escape from gene silencing in ICF syndrome: evidence for advanced replication time as a major determinant. Hum Mol Genet 2000; 9: 2575-2587.

12 Brun ME, Lana E, Rivals I et al: Heterochromatic genes undergo epigenetic changes and escape silencing in Immunodeficiency, centromeric instability, facial anomalies (ICF) syndrome. PLoS One 2011; 6: e19464.

13 Ehrlich M, Buchanan KL, Tsien F et al: DNA methyltransferase 3B mutations linked to the ICF syndrome cause dysregulation of lymphogenesis genes. Hum Mol Genet 2001; 10: 2917-2931.

14 Ehrlich M, Sanchez C, Shao C et al: ICF, an immunodeficiency syndrome: DNA methyltransferase $3 \mathrm{~B}$ involvement, chromosome anomalies, and gene dysregulation. Autoimmunity 2008; 41: 253-271.

15 Jin B, Tao Q, Peng J et al: DNA methyltransferase 3B (DNMT3B) mutations in ICF syndrome lead to altered epigenetic modifications and aberrant expression of genes regulating development, neurogenesis and immune function. Hum Mol Genet 2008; 17: 690-709.

16 Velasco G, Hubé F, Rollin J et al: Dnmt3b recruitment through E2F6 transcriptional repressor mediates germ-line gene silencing in murine somatic tissues. Proc Natl Acad Sci USA 2010; 107: 9281-9286.

17 Hassan KM, Norwood T, Gimelli G, Gartler SM, Hansen RS: Satellite 2 methylation patterns in normal and ICF syndrome cells and association of hypomethylation with advanced replication. Hum Genet 2001; 109: 452-462.

18 Michalet $\mathrm{X}$, Ekong R, Fougerousse $\mathrm{F}$ et al: Dynamic molecular combing: stretching the whole human genome for high-resolution studies. Science 1997; 277: 1518-1523.

19 Lengronne A, Pasero P, Bensimon A, Schwob E: Monitoring S phase progression globally and locally using BrdU incorporation in TK(+) yeast strains. Nucleic Acids Res 2001; 29: 1433-1442.

20 Grunau C, Sanchez C, Ehrlich M et al: Frequent DNA hypomethylation of human juxtacentromeric BAGE loci in cancer. Genes Chromosomes Cancer 2005; 43: 11-24.

21 Jackson DA, Pombo A: Replicon clusters are stable units of chromosome structure: evidence that nuclear organization contributes to the efficient activation and propagation of S phase in human cells. J Cell Biol 1998; 140: 1285-1295.

22 Conti C, Saccà B, Herrick J, Lalou C, Pommier Y, Bensimon A: Replication fork velocities at adjacent replication origins are coordinately modified during DNA replication in human cells. Mol Biol Cell 2007; 18: 3059-3067. 
23 Courbet S, Gay S, Arnoult N et al: Replication fork movement sets chromatin loop size and origin choice in mammalian cells. Nature 2008; 455: 557-560.

24 Bickmore WA, Carothers AD: Factors affecting the timing and imprinting of replication on a mammalian chromosome. J Cell Sci 1995; 108: 2801-2809.

25 Borgel J, Guibert S, Li Y et al: Targets and dynamics of promoter DNA methylation during early mouse development. Nat Genet 2010; 42: 1093-1100.

26 Ehrlich M, Jackson K, Weemaes C: Immunodeficiency, centromeric region instability, facial anomalies syndrome (ICF). Orphanet J Rare Dis 2006; 1: 2.

27 Tuck-Muller CM, Narayan A, Tsien F et al: DNA hypomethylation and unusual chromosome instability in cell lines from ICF syndrome patients. Cytogenet Cell Genet 2000; 89: 121-128.
28 Jefford CE, Irminger-Finger I: Mechanisms of chromosome instability in cancers. Crit Rev Oncol Hematol 2006; 59: 1-14

29 Rao VA, Conti C, Guirouilh-Barbat J et al: Endogenous gamma-H2AX-ATM-Chk2 checkpoint activation in Bloom's syndrome helicase deficient cells is related to DNA replication arrested forks. Mol Cancer Res 2007; 5: 713-724.

30 Sangrithi MN, Bernal JA, Madine $M$ et al: Initiation of DNA replication requires the RECQL4 protein mutated in Rothmund-Thomson syndrome. Cell 2005; 121 : 887-898.

31 Cadoret JC, Meisch F, Hassan-Zadeh V et al: Genome-wide studies highlight indirect links between human replication origins and gene regulation. Proc Natl Acad Sci USA 2008; 105: 15837-15842.

Supplementary Information accompanies the paper on European Journal of Human Genetics website (http://www.nature.com/ejhg) 